\title{
Comunicación
}

\section{Uso del Azufre Precipitado en Petrolato para el Tratamiento Tópico de la Sarna Knemidocóptica (Knemidocoptessp) en Dos Gallos}

\author{
Use of Precipitated Sulphur in Pretrolatum for the Topical Treatment of \\ KNemidocoptic Mange (Knemidocoptes sp) in Two Roosters
}

Dalmiro Cazorla Perfetti' ${ }^{1,2}$, Pedro Morales Moreno ${ }^{1}$

\section{Resumen}

Se presentan los resultados de un ensayo clínico para evaluar la aplicación tópica del azufre precipitado en petrolato (10\%) en el tratamiento de lesiones cutáneas podales crónicas de sarna knemidocóptica en dos gallos (Gallus gallus domesticus) en la ciudad de Coro, estado Falcón, Venezuela. El diagnóstico se realizó mediante examen clínico y de laboratorio, tomándose muestras de las lesiones dérmicas por raspado, procesadas con $\mathrm{KOH}(10 \%)$ y observadas al microscopio. El tratamiento topical fue realizado cada 3 días durante 1 mes. La evaluación del tratamiento se hizo mediante observación de la evolución y raspado de las lesiones. La valoración de los efectos adversos se hizo mediante observación clínica. Los dos gallos se recuperaron de las lesiones sin observarse efectos secundarios. La mezcla de azufre precipitado en petrolato aparece como una alternativa simple y de bajo costo para el tratamiento tópico de la sarna knemidocóptica en Gallus gallus domesticus.

Palabras clave: sarna knemidocóptica, gallos, azufre, petrolato, tratamiento tópico

\section{Abstract}

The short communication shows the results of a clinical trial to evaluate the topical application of precipitated sulphur in petrolatum $(10 \%)$ in the treatment of chronic feet skin lesions of knemidocoptic mange in two roosters in Coro city, Falcon state, Venezue-

\footnotetext{
${ }^{1}$ Laboratorio de Entomología, Parasitología y Medicina Tropical (L.E.P.A.M.E.T.), Centro de Investigaciones Biomédicas (C.I.B.), Universidad Nacional Experimental «Francisco de Miranda»(UNEFM), Estado Falcón, Venezuela

${ }^{2}$ E-mail: lutzomyia@hotmail.com
}

Recibido: 10 de setiembre de 2015

Aceptado para publicación: 18 de febrero de 2016 
la. The diagnosis was made by clinical and laboratory examination of scrapings of cutaneous lesions processed with $\mathrm{KOH} 10 \%$ and microscopically observed. The topical treatment was performed every 3 days for 1 month. The evaluation of the treatment was done by observing the evolution of the lesions and scraping of the knemidocoptic lesions and the assessment of the side-effects was done by clinical observation. Both birds recovered from the injuries without side effects. The mixture of precipitated sulfur in petrolatum appears as a simple and inexpensive alternative for the topical treatment of knemidocoptic mange in Gallus gallus domesticus.

Key words: knemidocoptic mange, cocks, sulphur, petrolatum, topical treatment

\section{INTRODUCCIÓN}

La sarna knemidocóptica es una patología dérmica muy importante que afecta a las aves, tanto domésticas como silvestres, especialmente a individuos de las órdenes Galliformes, Passeriformes y Psitaciformes de las regiones tropicales, incluyendo Venezuela (Meléndez y Yépez, 1977; Mironov et al., 2005; Bruno y Albuquerque, 2008). Se le conoce con el nombre común de «sarna de las patas», «roña de las patas», «escama de las patas», «patas calcáreas», «patas sarnosas» o «patas elefantiásicas» (Meléndez y Yépez, 1977; Quiroz Romero, 1999).

Esta ectoparasitosis es causada por varias especies de ácaros (Arachnida: Acarina) del género Knemidocoptes (Sarcoptiformes, Epidermoptidae, Knemidokoptinae). Los ácaros tienen forma circular, con patas cortas y cutícula sin espinas y estriada, a través de la cual respiran, por lo que se les considera del suborden Astigmata (Fain y Elsen, 1967; Mironov et al., 2005; Dabert et al., 2013). En el caso de las Galliformes, especialmente en gallinas domésticas (Gallus gallus domesticus), se les encuentra bajo las escamas de las patas, donde abren surcos o túneles (ácaro arador) para alimentarse de queratina (Quiroz Romero, 1999; Wall y Shearer, 2001; Bruno y Albuquerque, 2008), causando irritación, prurito y exudaciones serosas, llegando a la aparición masiva de costras e hinchazón de las patas. En infestaciones masivas y descuidadas, debido a la hiperqueratosis, puede ocurrir la deformación del área podal con postración del animal y artritis, emaciación y muerte por inanición (Quiroz Romero, 1999; Wall y Shearer, 2001; Bruno y Albuquerque, 2008).

En el tratamiento quimioterapéutico de la sarna se ha empleado durante mucho tiempo diversos fármacos de aplicación tópica, incluyendo la rotenona, benzoato de bencilo, ortofenilfenol, crotamitón, lindano, moxidectina e ivermectina (Chauhan y Roy, 1996; Quiroz Romero, 1999; Wall y Shearer, 2001). Algunos de estos acaricidas pueden tener efectos tóxicos, especialmente en aves pequeñas, y su eficacia está limitada para ciertos estadios de desarrollo del ácaro; asimismo, el costo de adquisición de algunos puede ser oneroso para la población rural campesina (Chauhan y Roy, 1996; Quiroz Romero, 1999; Marín-Gómez y Benavides-Montaño, 2007).

El conocimiento de la acción acaricida del azufre es milenario (Lin et al., 1988) y el azufre precipitado en petrolato es utilizado tradicionalmente para el tratamiento de la sarna knemidocóptica (Chauhan y Roy, 1996). Tiene la ventaja de su bajo costo y sus componentes son de amplia disponibilidad en los mercados farmacéuticos de los países en desarrollo. Sin embargo, no se dispone de estudios controlados en Venezuela que avalen la utilización de los ungüentos azufrados contra los ácaros del género Knemidocoptes. En el presente estudio, se presentan los resultados de la aplicación tópica del azufre 
precipitado en petrolato en dos gallos con sarna knemidocóptica podal.

\section{Materiales y Métodos}

Las observaciones y hallazgos se realizaron en dos gallos blancos adultos de tipo genético desconocido. Uno de 4 años y 6 meses con $3.4 \mathrm{~kg}$ de peso vivo (gallo 1 ) y el otro de 4 años y 2 meses con $2.7 \mathrm{~kg}$ de peso vivo (gallo 2). Las aves estaban en jaulas metálicas $(1.5 \times 1.1 \times 0.8 ; 1.2 \times 0.6 \times 0.7 \mathrm{~m}$, respectivamente) con piso de arena. En el manejo y crianza de los gallos se siguieron las normas internacionales y nacionales en el cuidado y uso de animales de laboratorio (FONACIT, 2008).

Las aves se criaban en las áreas adyacentes al Laboratorio de Entomología, Parasitología y Medicina Tropical (LEPAMET) de la Universidad Nacional Experimental «Francisco de Miranda», en la ciudad de Coro, capital del estado Falcón, Venezuela. Estas aves se utilizan rutinariamente para la alimentación de las colonias de triatominos (Rhodnius prolixus, Triatoma maculata) del insectario del LEPAMET, utilizados en docencia, experimentación y xenodiagnóstico en animales y personas.

Los gallos presentaban lesiones hiperqueratósicas en ambas patas y uñas, con costras o escamas epidérmicas hipertróficas de varios tamaños, rugosas y porosas de color gris-amarillento en la parte dorsal y ventral de los dedos, así como en la parte distal de la región tarso-metatarso (Figuras 1A, 1D). Debido al descuido o negligencia del personal a cargo, las lesiones podales de desarrollaron por espacio de 8 a 10 meses, llevando al agrandamiento de los miembros. Ambos animales se mostraban inquietos y se picoteaban frecuentemente las patas, lo que era un indicio del prurito que ocasionaba la acción del ácaro. El gallo 2 presentaba dificultades para movilizarse.
El diagnóstico clínico de la sarna knemidocóptica fue confirmado en el laboratorio. Se hizo un raspado de la región afectada, el material epidérmico fue colocado sobre una lámina portaobjeto y se mezcló con $\mathrm{KOH}$ al $10 \%$, se cubrió con una laminilla cubreobjeto y se observó en el microscopio óptico (10X) (Axiostar Plus, Carl Zeiss, Alemania). Se observaron huevos, estadios preimaginales y adultos de Knemidocoptes $\mathrm{sp}$, que fueron identificados por sus características morfo-taxonómicas (Fain y Elsen, 1967; Bruno y Albuquerque, 2008; Dabert et al., 2013).

Para el tratamiento tópico se utilizó una mezcla de azufre precipitado en petrolato (vaselina) al 10\%, preparado en el LEPAMET. El ungüento se aplicó en las lesiones podales cada 3 días durante 1 mes. Adicionalmente, se siguió como norma de higiene el cambio semanal de la arena del piso de las jaulas y el lavado con agua tibia de las patas antes de la aplicación del tratamiento.

Se hizo una evaluación clínica diaria durante 40 días, mediante observación de la evolución de las lesiones cutáneas asociadas a la sarna knemidocóptica y de la conducta de los animales (intranquilidad, picoteo de las lesiones); asimismo, se hizo un raspado de las zonas lesionadas al mes de aplicada la primera dosis para la evaluación ectoparasitológica. La tolerancia hacia la droga se evaluó por examinación clínica de los posibles efectos secundarios que eventualmente pueden inducir los componentes del ungüento, especialmente sobre la piel y la conducta de las aves.

\section{Resultados y Discusión}

El análisis ectoparasitológico confirmó la presencia de los ácaros del género Knemidocoptes en las lesiones podales de ambas aves (Fain y Elsen, 1967; Mironov et al., 2005; Dabert et al. 2011). Las aves respondieron adecuadamente al tratamiento. 


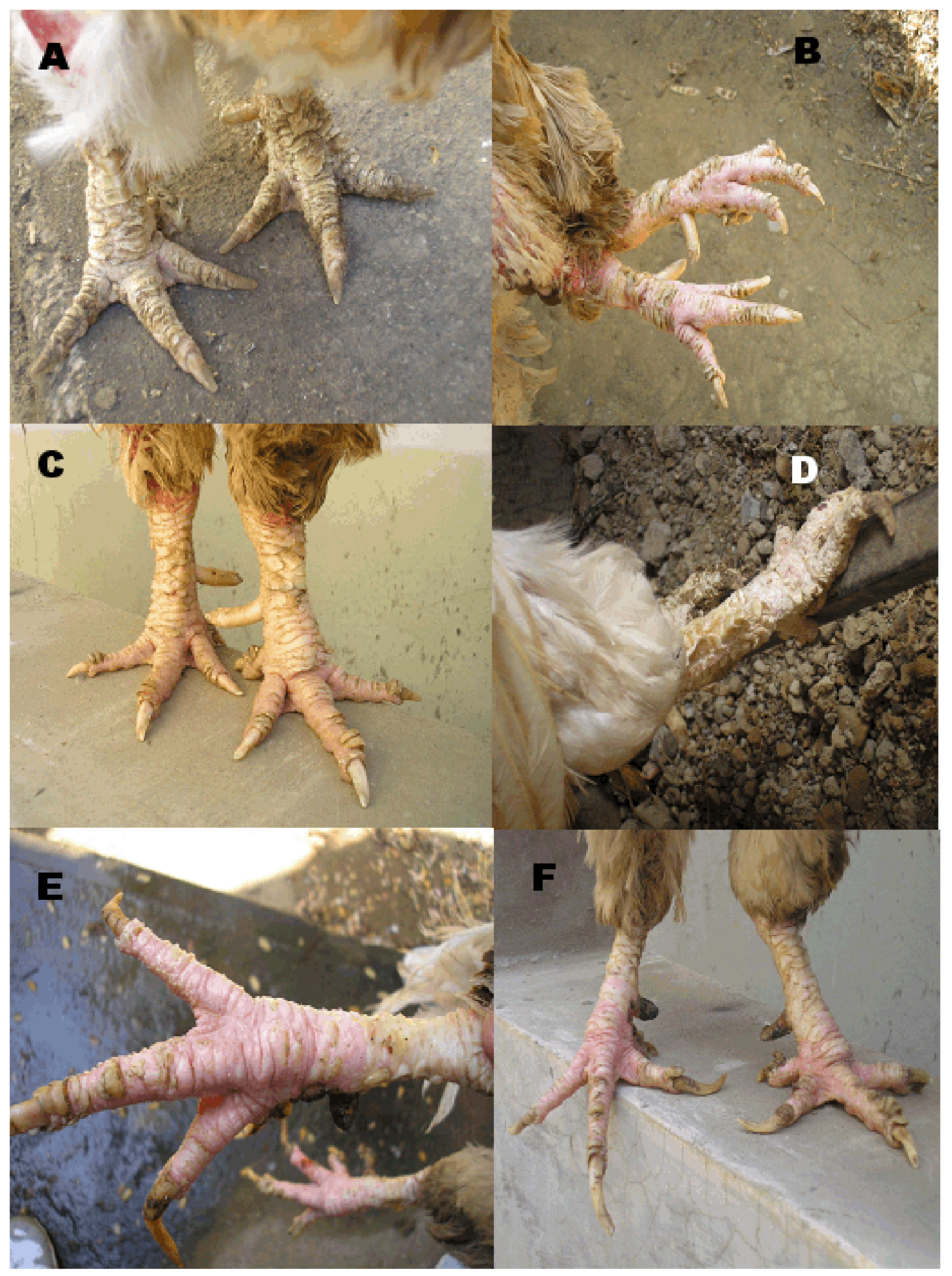

Figura 1. Gallo 1: A) Patas antes del tratamiento; B) 15 días de iniciado el tratamiento ( $5^{\mathrm{a}}$ dosis); C) un mes de iniciado el tratamiento ( $10^{\mathrm{a}}$ dosis). Gallo 2: D) Patas antes del tratamiento; E) 15 días de iniciado el tratamiento ( $5^{\text {a }}$ dosis); F) un mes de iniciado el tratamiento $\left(10^{\mathrm{a}}\right.$ dosis $)$ 
A los 5 días de haberse aplicado la primera dosis de azufre precipitado en petrolato al $10 \%$ empezaron a desprenderse las primeras escamas. A los 9-10 días dejaron de picotearse las patas y de permanecer inquietos; y a los 15 días la mayoría de las costras afectadas se habían desprendido, y la piel mostraba una turgencia que se puede considerar como normal, con aparición de nuevas escamas epidérmicas y de coloración rosácea (Figuras 1B, 1E). Al mes de iniciado el tratamiento, las aves se encontraban clínica y ectoparasitológicamente curadas (Figuras 1C, 1F). El gallo 2 llegó a recuperar totalmente su movilidad. Asimismo, es importante notar que no se observaron efectos secundarios por el tratamiento.

La aplicación tópica de una droga en un área delimitada es más segura que la vía sistémica, debido a que se puede suministrar mayores concentraciones del fármaco con menor riesgo de efectos secundarios indeseables (Urroz, 2000). El exceso de azufre inorgánico generado en el metabolismo hepático o renal se elimina por la orina en forma de sulfatos, por lo que se le considera un elemento seguro de baja toxicidad (Lee, 2013). Esto hace del azufre un compuesto muy seguro frente a otros acaricidas de origen químico (por ejemplo, los organofosforados y piretroides), que se acumulan en los tejidos del animal y el medio ambiente, siendo potencialmente tóxicos para el ser humano, y cuyo uso indiscriminado trae como consecuencia la aparición de poblaciones de ácaros resistentes (Marangi et al., 2012).

El efecto queratolítico del azufre es conocido, especialmente cuando se aplica a dosis superiores del $5 \%$, pues favorece la formación de sulfuro de hidrógeno que disuelve el estrato córneo. Esto permite que se desprendan las escamas y, por consiguiente, los ácaros. Además, al disolver los surcos acarinos, el azufre actuaría con mayor facilidad sobre los ácaros (Lin et al., 1988). Asimismo, debe tomarse en consideración el efecto de aniquilamiento por oclusión y sofocación que ocasiona la vaselina a cortar el suministro de oxígeno a este tipo de ácaros (Astigmata), así como a otros tipos de ectoparásitos que poseen la cutícula muy fina (Jacobson y Abel, 2007).

El azufre y la vaselina se encuentran ampliamente disponibles en el mercado y son de bajo costo (azufre: $20 \mathrm{~g}$ por US\$ 3.16 ; vaselina: $100 \mathrm{~g}$ por US\$7.50).

\section{Literatura Citada}

1. Bruno S, Albuquerque D. 2008. Ocorrência e tratamento de sarna knemidocóptica (Knemidokoptes sp.) em aves de companhia atendidas na Faculdade de Veterinária da Universidade Federal Fluminense, RJ. Ciênc Rural 38: 1472-1475. doi: 10.1590/ S0103-84782008000500046

2. Chauhan H, Roy S. 1996. Poultry diseases, diagnosis and treatment. $2^{\text {nd }} e d$. India: New Age International. 430 p.

3. Dabert J, Mihalca A, Sándor A. 2011. The first report of Knemidocoptes intermedius Fain et Macfarlane, 1967 (Acari: Astigmata) in naturally infected European birds. Parasitol Res 109: 237 240. doi: 10.1007/s00436-011-2390-8

4. Dabert J, Dabert M, Gal A, Miclãu ${ }^{o}$ V, Mihalca A, Sándor A. 2013. Multidisciplinary analysis of Knemidocoptes jamaicensis parasitising the Common Chaffinch, Fringilla coelebs: proofs for a multispecies complex? Parasitol Res 112: 2373-2380. doi: 10.1007/s00436-013-3402-7

5. Fain A, Elsen P. 1967. Les acariens de la famille Knemidokoptidae, producteurs de la gale chez les oiseaux (Sarcoptiformes). Acta Zool Pathol Antverp 45: 3-145.

6. [FONACIT] Fondo Nacional de Ciencia y Tecnología. 2008. Código de bioética y bioseguridad [Internet]. Disponible en: http://www.ciens.ucv.ve: 8080/generador/sites/biolanimlab/archivos/codigo_fonacit_2008.pdf 
7. Jacobson C, Abel E. 2007. Parasitic infestations. J Am Acad Dermatol 56: 1026-1043. doi: 10.1016/j.jaad.2006. 10.963

8. Lee M. 2013. Ingesta: los nutrientes y su metabolismo. En: Mahan K, EscottStump S, Raymond J (eds). Krause dietoterapia. $13^{\mathrm{a}}$ ed. Amsterdam, Holanda: Elsevier. p 104-105.

9. Lin A, Reimer R, Carter D. 1988. Sulfur revisited. J Am Acad Dermatol 18: 553-558. doi: 10.1016/S01909622(88)70079-1

10. Lofgren E. 2014. The backyard chicken bible: the complete guide to raising chickens. Wisconsin, USA: Living Ready Books. 240 p.

11. Marangi M, Morelli V, Pati S, Camarda A, Cafiero M, Giangaspero A. 2012. Acaricide residues in laying hens naturally infested by red mite Dermanyssus gallinae. PLoS One 7(2): e31795. doi: 10.1371/journal.pone. 0031795

12. Marín-Gómez S, Benavides-Montaño J. 2007. Parásitos en aves domésticas
(Gallus domesticus) en el noroccidente de Colombia. Vet Zootec 1: 43-51.

13. Meléndez R, Yépez M. 1977. Estudio sobre ácaros parásitos. I. Acariasis de las aves de corral en Venezuela, con especial referencia a la especie Dermatophagoides scheremetewskyi. Vet Trop 2:3-9.

14. Mironov S, Bochkov A, Fain A. 2005. Phylogeny and evolution of parasitism in feather mites of the families Epidermoptidae and Dermationidae (Acari: Analgoidea). Zool Anz 243:155179. doi: 10.1016/j.jcz.2004.10.001

15. Quiroz Romero H. 1999. Parasitología y enfermedades parasitarias de animales domésticos. México DF: Ed Limusa. $876 \mathrm{p}$.

16. Serra-Baldrich E. 1996. Azufre, compuestos y uso. Act Dermatol 35: 163170.

17. Urroz C. 2000. Farmacología y manejo de productos veterinarios. Principios básicos. Costa Rica: EUNED. 196 p.

18. Wall R, Shearer D. 2001. Veterinary ectoparasites: biology, pathology and control. $2^{\text {nd }}$ ed. UK: Blackwell Science. 304 p. 